\title{
Shape-Induced Anisotropy in Antidot Arrays From Self-Assembled Templates
}

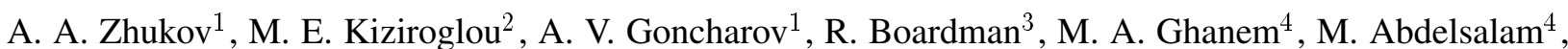 \\ V. Novosad ${ }^{5}$, G. Karapetrov ${ }^{5}$, X. Li ${ }^{2}$, H. Fangohr ${ }^{3}$, C. H. de $\operatorname{Groot}^{2}$, P. N. Bartlett ${ }^{4}$, and P. A. J. de Groot ${ }^{1}$ \\ ${ }^{1}$ School of Physics and Astronomy, University of Southampton, Southampton SO17 1BJ, U.K. \\ ${ }^{2}$ School of Electronics and Computer Science, University of Southampton, Southampton SO17 1BJ, U.K. \\ ${ }^{3}$ School of Engineering Sciences, University of Southampton, Southampton SO17 1BJ, U.K. \\ ${ }^{4}$ School of Chemistry, University of Southampton, Southampton SO17 1BJ, U.K. \\ ${ }^{5}$ Material Science Division, Argonne National Laboratory, Argonne, IL 60439 USA
}

\begin{abstract}
Using self-assembly of polystyrene spheres, well-ordered templates have been prepared on glass and silicon substrates. Strong guiding of self-assembly is obtained on photolithographically structured silicon substrates. Magnetic antidot arrays with three-dimensional architecture have been prepared by electrodeposition in the pores of these templates. The shape anisotropy demonstrates a crucial impact on magnetization reversal processes.
\end{abstract}

Index Terms-Electrodeposition, magnetic hysteresis, nanotechnology, self-assembly, silicon.

\section{INTRODUCTION}

$\mathbf{T}$ HE DEMAND for higher magnetic recording densities is stimulating intense research activity into magnetism on submicrometer-length scales. The main requirement for commercially viable products is an efficient and low-cost preparation process. Methods based on templates formed by the self-assembly of colloidal particles have been considered for various applications such as photonic materials [1], microchip reactors [2], and biosensors [3]. In this paper, we use ordered templates for the electrodeposition of various magnetic materials. This technique offers new opportunities, which are not easily realized by standard lithographic methods, and which allow us to create magnetic nanostructures with three-dimensional (3-D) architectures on a broad range of length scale, $20-1000 \mathrm{~nm} .{ }^{1}$ To address limitations of self-assembly (a lack of long-range order and control of array directions), we are employing hybrid techniques using well-established Si technology to guide and confine self-assembly templates (e.g., [4]).

\section{EXPERIMENTAL PROCEDURE}

Using slow evaporation of a colloidal water suspension containing $1 \mathrm{wt}$ \% of latex spheres, well-ordered templates have been self-assembled on glass substrates with buffer layers of sputtered $\mathrm{Cr}(10 \mathrm{~nm})$ and $\mathrm{Au}(200 \mathrm{~nm})$ or on HF-treated $\mathrm{Si}$. These templates have been used as moulds to prepare nanoporous magnetic ( $\mathrm{Ni}, \mathrm{Co}, \mathrm{Fe}$, and $\mathrm{Ni}_{50} \mathrm{Fe}_{50}$ ) structures by electrodeposition methods [5].

Magnetic measurements were performed using a superconducting quantum interference device (SQUID), a vibrating sample magnetometer (VSM), and a magnetooptical Kerr effect (MOKE) rig. Most measurements have been performed at room

Digital Object Identifier 10.1109/TMAG.2005.854734

$120 \mathrm{~nm}$ is the smallest available size of polystyrene spheres. temperature with the magnetic field applied in parallel to the plane of the film. Magnetic structures have been imaged using magnetic force microscopy (MFM) by a Digital Instruments 3000 scanning probe microscope in lift-mode with a fly height of $100 \mathrm{~nm}$ and a standard low-moment ferromagnetic tip.

\section{RESULTS AND DISCUSSION}

\section{A. Guided Self-Assembly and Electrodeposition on Structured Silicon}

To study confinement effects and to guide ordering in defined directions, we have used a scaffolding method for guided self-assembly by various lithographically prepared patterns on the surface of a Si substrate. For this purpose, a 250-nm thermal $\mathrm{SiO}_{2}$ layer was grown on the $\mathrm{Si}$ wafers and different patterns (stripes, squares, triangles, circles, and rings) were defined by photolithography. Subsequently, the wafers were immersed in a 20:1 buffered HF solution to etch the oxide and to expose $\mathrm{Si}$ areas of different shapes and sizes ranging from 1.5 to $50 \mu \mathrm{m}$. Then, the wafers were sawed in pieces, RCA (Radio Corporation of America) process cleaned [6], and dipped in 20:1 buffered HF prior to latex sphere self-assembly. After the self-assembly, the wafers with sphere templates were dipped again in 20:1 buffered HF. The spheres adhesion, presumably governed by van der Waals forces [7], is sufficient to withstand this process. As we can see from Fig. 1, the template is formed on the structured areas of $\mathrm{Si}$, and essentially no spheres can be observed in the region of $\mathrm{SiO}_{2}$ (dark gray surface). The template structure demonstrates well-pronounced guiding with the side of a triangular unit cell parallel to the stripe direction. $\mathrm{Next}$, Ni films were grown on the conducting $\mathrm{Si}$ areas by electrodeposition. To improve the smoothness, pulsed electrochemical pretreatment was used. Finally, the spheres were dissolved in tetrahydrofuran.

The resulting magnetic antidot array is the inverse of the template and also demonstrates well-guided behavior in the case of 


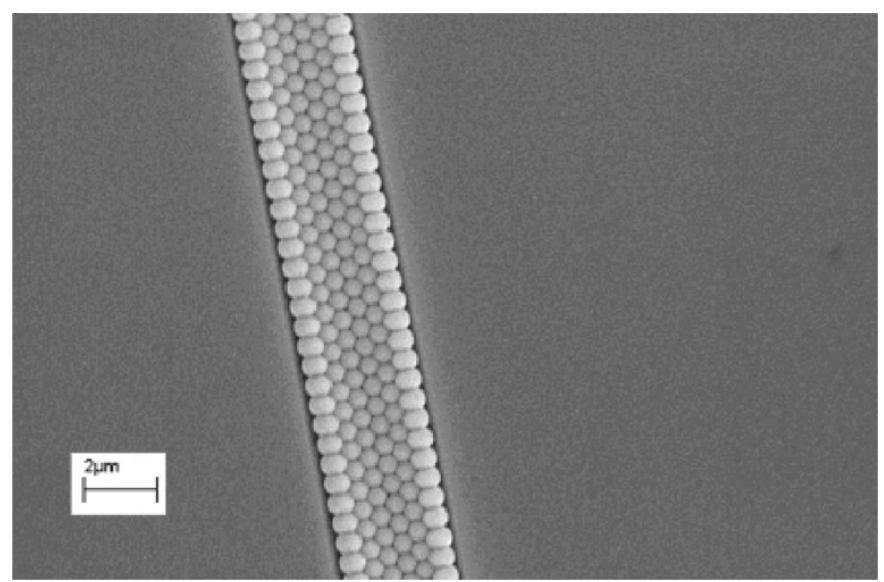

Fig. 1. SEM image of 500-nm-diameter spheres self-assembled in a stripe grove of patterned $\mathrm{Si}-\mathrm{SiO}_{2}$ substrate.

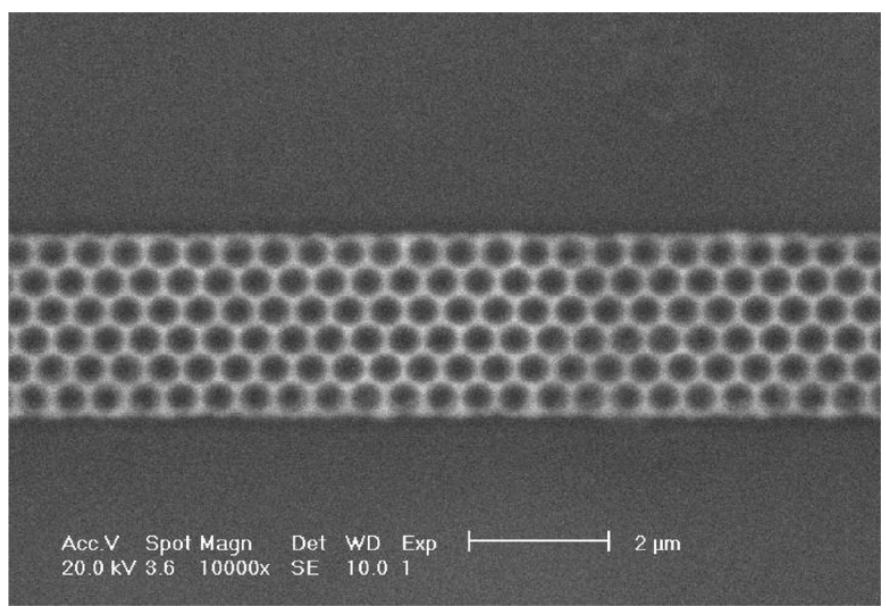

Fig. 2. SEM image of nanostructured $\mathrm{Ni}$ film prepared by guided self-assembly of 500-nm spheres on a patterned $\mathrm{Si}-\mathrm{SiO}_{2}$ substrate.

stripes (Fig. 2). For square $\mathrm{Si}_{-} \mathrm{SiO}_{2}$ microstructures the self-assembly was guided along one of the square sides. No guiding effect was observed for right-angle triangles, circles and rings. This probably results from competition between different directions favored by their shape.

\section{B. Magnetization Reversal and Shape Anisotropy}

The template nanostructuring strongly influences the magnetic properties (e.g., [8]). The 3-D architecture results in behavior resembling that of multilayers with alternating hard and soft layers: the coercive field $B_{c}$ has been found to oscillate with the thickness of the film $t_{f}$ (Fig. 3). $B_{c}$ reaches a maximum for the case when the top surface of the film is near the void centers. For layers with complete spherical voids, the coercive field approaches a minimum. These observations suggest that the points where the spheres touch play an important role in domain-wall pinning and hence the coercivity [9]. Our results demonstrate that 3-D patterned magnetic materials are prototypes of a new class of geometrical multilayer structures in which the layering is due to local shape effects rather then compositional differences.

To investigate the local magnetic structures of the templated films, we employed MFM complemented by micromagnetic

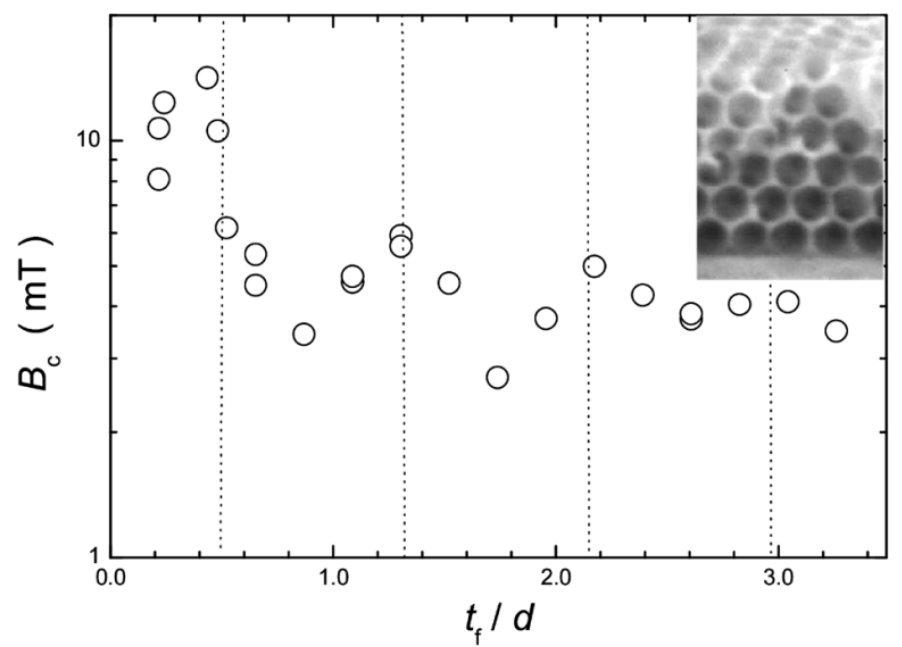

Fig. 3. Coercive field for $\mathrm{Ni}_{50} \mathrm{Fe}_{50}$ films with different thickness $t_{f}$ prepared using 550-nm spheres. Dashed lines show the positions of sphere centers for each layer in a close packed structure. Inset demonstrates layered 3-D architecture for a thick film.

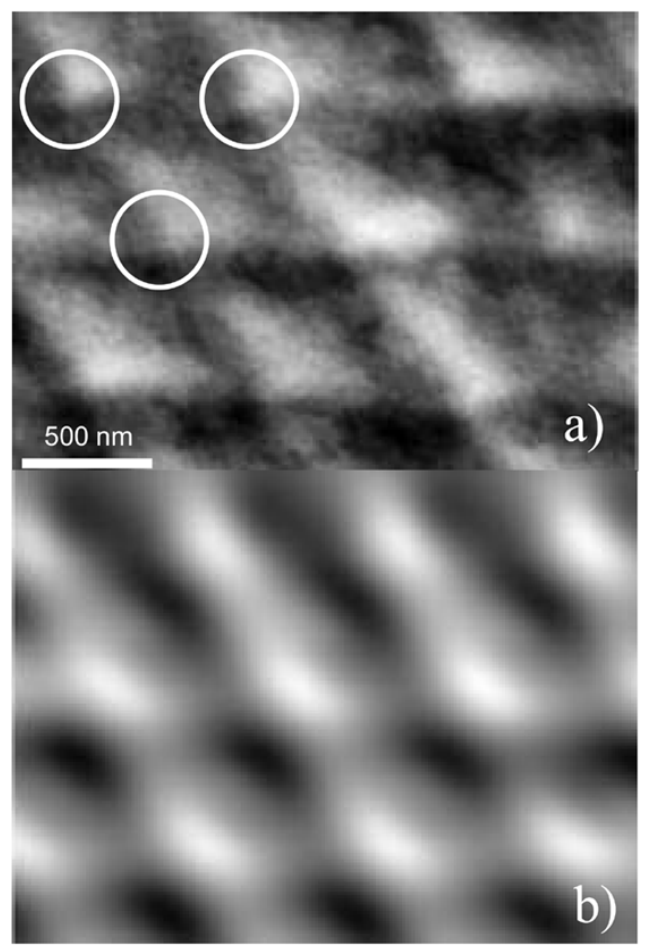

Fig. 4. MFM images measured for Co antidot film with $d=700 \mathrm{~nm}$ and (a) $t_{f}=100 \mathrm{~nm}$ and from (b) micromagnetic modeling. White circles indicate hole positions.

modeling. Results are shown in Fig. 4(a) for a relatively thin $(100 \mathrm{~nm})$ Co film with sphere diameter $d=700 \mathrm{~nm}$ in the remanent state. These images reveal ordered rhombic magnetic patterns associated with the antidot array. Magnetic structures for two-dimensional (2-D) antidot arrays were calculated using micromagnetic modeling with the OOMMF software suite [10]. Parameters in the computation were chosen in accordance with the known values for Co [11], cell size was $2 \mathrm{~nm}$, and hole diameters of $0.4 d$ correspond to the top of the film. The MFM signal was modeled by computing the second derivative of the dipolar field produced by the film [12] at a distance of the fly 


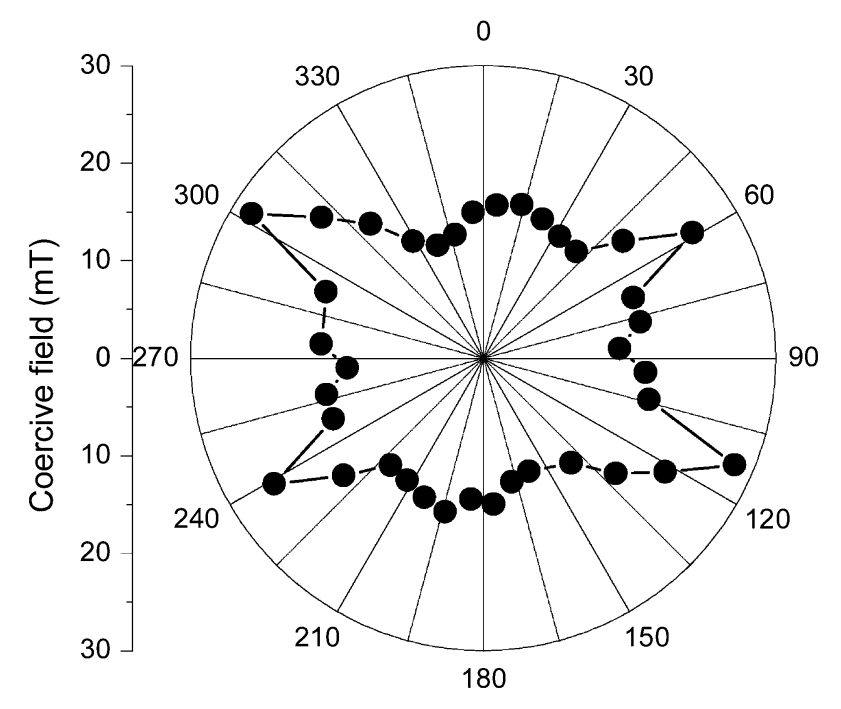

Fig. 5. Anisotropy of coercive field in plain Ni antidot array prepared from 900-nm spheres. Zero angle corresponds to the direction of unit vector of array.

height above the film [Fig. 4(b)]. The picture obtained from 2-D micromagnetic simulations is similar to the results of MFM.

We have measured angular dependences of a coercive field using MOKE to reveal the influence of shape anisotropy produced by nanostructuring (Fig. 5). The obtained results reveal pronounced six-fold symmetry corresponding to the hexagonal configuration of the nanostructured antidot array. The small distortion along $0^{\circ}$ direction is probably caused by film texturing. The micromagnetic simulations [10] qualitatively reproduce the experimental observations with sharp maxima along the bysectors of unit cell triangles.

\section{CONCLUSION}

Using self-assembly from colloidal suspensions of polystyrene latex spheres, we have prepared well-ordered nanostructured templates. We present results of electrochemical deposition of magnetic metals and alloys in the voids of these templates, enabling the fabrication of magnetic antidot nanostructures with 3-D architectures. We find large changes in the coercive field, dominated by the effects of the nanoscale shapes. These 3-D antidot nanostructures reveal oscillations of the coercive field in the dependence on film thickness. Varying the parameters in the preparation (sphere diameter, film thickness, and composition) allows us to produce materials with predetermined magnetic parameters. To address limitations of self-assembly (lack of long-range order and control of array directions), we are employing hybrid techniques using well-established Si technology to guide and confine self-assembly templates. The template growth technique has the potential to lead to a low-cost preparation method for submicron patterned magnetic materials.

\section{ACKNOWLEDGMENT}

This work was supported in part by the U.K. Engineering and Physical Sciences Research Council under Grant GR/S13200/01 and Grant EP/C511 786/1.

\section{REFERENCES}

[1] Y. A. Vlasov, X.-Z. Bo, J. C. Sturm, and D. J. Norris, "On-chip natural assembly of silicon photonic bandgap crystals," Nature, vol. 414, pp. 289-293, Nov. 2001.

[2] H. Gau, S. Herminghaus, P. Lenz, and R. Lipowsky, "Liquid morphologies on structured surfaces: From microchannels to microchips," Science, vol. 283, pp. 46-49, Jan. 1999.

[3] O. D. Velev and E. W. Kaler, "In situ assembly of colloidal particles into miniaturized biosensors,” Langmuir, vol. 15, pp. 3693-3698, May 1999.

[4] J. Y. Cheng, C. A. Ross, E. L. Thomas, H. I. Smith, and G. J. Vancso, "Templated self-assembly of block copolymers: Effect of substrate topography," Adv. Mater., vol. 15, pp. 1599-1602, Oct. 2003.

[5] P. N. Bartlett, M. A. Ghanem, I. S. El Hallag, P. de Groot, and A. Zhukov, "Electrochemical deposition of macroporous magnetic networks usin colloidal templates," J. Mater. Chem., vol. 13, pp. 2596-2602, Aug. 2003.

[6] W. Kern and J. Vossen, Thin Film Processes. New York: Academic, 1978, ch. V-1.

[7] J. Israelachvili, Intermolecular Surface Forces, 2nd ed. London, U.K.: Academic, 1992, pp. 176-177.

[8] A. A. Zhukov, A. V. Goncharov, P. A. J. de Groot, P. N. Bartlett, M. A. Ghanem, H. Küpfer, R. J. Pugh, and G. J. Tomka, "Patterned magnetic media from self-assembly template methods," in IEE Proc.-Sci. Meas. Technol., vol. 150, Sep. 2003, pp. 257-259.

[9] A. A. Zhukov, M. A. Ghanem, A. V. Goncharov, R. Boardman, V. Novosad, G. Karapetrov, H. Fangohr, P. N. Bartlett, and P. A. J. de Groot, Oscillatory thickness dependence of the coercive field in magnetic 3d anti-dot arrays. \#cond-mat/0 406091.

[10] "Object oriented micromagnetic framework" [Online]. Available: http:// math.nist.gov/oommf/.

[11] R. Skomski and J. M. D. Coey, "Permanent magnetism," Ins. Phys., 1999.

[12] J. M. Garcia, A. Thiaville, J. Miltat, K. J. Kirk, J. N. Chapman, and F. Alouges, "Quantitative interpretation of magnetic force microscopy images from soft patterned elements," Appl. Phys. Lett., vol. 79, pp. 656-658, Jul. 2001.

Manuscript received February 7, 2005. 